\title{
CINT Referees 2015-2016
}

We at Contexto Internacional would like to extend our appreciation to each peer reviewer who have kindly contributed with thoughtful, helpful and rigorous comments to submitted manuscripts and for contributing to the scientific and editorial improvement of our journal in the last couple of years. We would also like to thank the members of CINT's Communication Team who have assisted the journal in its internationalization and outreach efforts. By generously sharing their time, expertise and insights, peer reviewers and CommTeam members have played an essential role in the advancement of CINT's mission, that of encouraging and promoting the development of International Relations scholarship in/of/from the Global South for broader international audiences.

Ajay Skaria, University of Minnesota (United States of America)

Alcides Vaz, Universidade de Brasília (Brazil)

Alejandro Simonoff, Universidad Nacional de La Plata (Argentina)

Alexandra Mello, Pontifícia Universidade Católica do Rio de Janeiro (Brazil)

Alexandre Cunha Leite, Universidade Estadual da Paraíba (Brazil)

Alexandre Fuccille, Universidade Estadual Paulista (Brazil)

Alexandre Queiroz Guimarães, Fundação João Pinheiro (Brazil)

Alice Amorim, Gestão de Interesse Público (Brazil)

Ana Flávia Granja e Barros, Universidade de Brasília (Brazil)

Ana Garcia, Universidade Federal Rural do Rio de Janeiro (Bazil)

Ana Paula Tostes, Universidade do Estado do Rio de Janeiro (Brazil)

Ananda Abeysekara, Virginia Polytechnic Institute and State University (United States of America)

André de Mello e Souza, Universidade Federal do Estado do Rio de Janeiro (Brazil)

André Luiz Coelho Souza, Universidade Federal do Estado do Rio de Janeiro (Brazil)

André Reis Silva, Universidade Federal do Rio Grande do Sul (Brazil)

Andrea Bianculli, Institut Barcelona d'Estudis Internacionals (Spain)

Andrea Ribeiro Hoffmann, Pontifícia Universidade Católica do Rio de Janeiro (Brazil)

Andrea Pacheco Pacifico, Universidade Estadual da Paraíba (Brazil)

Andreas Feldmann, University of Illinois-Chicago (United States of America)

Anna Stavrianakis, University of Sussex (United Kingdom)

Anthony Turton, University of Pretoria (South Africa)

Antonio Jorge Ramalho da Rocha, Universidade de Brasília (Brazil)

Antonio Josino, Universidade de São Paulo (Brazil)

Antonio Ruy de Almeida Silva, Escola Superior de Guerra (Brazil) 
Arlene Tickner, Universidad de los Andes (Chile)

Arthur Coelho, Universidade Federal do Rio Grande do Sul (Brazil)

Áureo de Toledo Gomes, Universidade Federal de Uberlandia (Brazil)

Bruno Ayllón Pino, Universidad Complutense de Madrid (Spain)

Bruno Borges, Universidade do Estado do Rio de Janeiro (Brazil)

Bruno Magalhães, Pontifícia Universidade Católica do Rio de Janeiro (Brazil)

Carlos Aurelio Faria, Pontifícia Universidade Católica de Minas Gerais (Brazil)

Carlos Frederico Gama, Universidade Federal do Tocantins (Brazil)

Catia Gregoratti, Lund University (Sweden)

Christian Edward Cyril Lynch, Universidade Estadual do Rio de Janeiro (Brazil)

Cintia Quiliconi, Facultad Latinoamericana de Ciencias Sociales Sede Ecuador (Ecuador)

Claudia Fuentes, Pontifícia Universidade Católica do Rio de Janeiro (Brazil)

Cristian Cantir, Oakland University (United States of America)

Cristiano Mendes, Pontifícia Universidade Católica de Minas Gerais (Brazil)

Cristina Inoue, Universidade de Brasília (Brazil)

Cristina Rojas, Carleton University (Canada)

Daniel Aragão, Universidade Federal da Bahia (Brazil)

Daniel Castelan, Universidade Federal de Santa Catarina (Brazil)

Danilo Marcondes, Pontifícia Universidade Católica do Rio de Janeiro (Brazil)

David Blaney, Macalester College (United States of America)

David Chandler, University of Westminster (United Kingdom)

David Criekemans, Universiteit Antwerpen (Belgium)

Dawisson Lopes, Universidade Federal de Minas Gerais (Brazil)

Deisy Ventura, Universidade de São Paulo (Brazil)

Detlef Nolte, German Institute of Global and Area Studies (Germany)

Diana Thomaz, Wilfrid Laurier University (Canada)

Diego Acosta Acarazo, University of Bristol (United Kingdom)

Eduardo Viola, Universidade de Brasília (Brazil)

Elif Kalaycioglu, University of Minnesota (United States of America)

Elke Krahmann, Universität Witten/Herdecke (Germany)

Elsa Cardozo, Universidad Central de Venezuela (Venezuela)

Ernesto Vivares, Facultad Latinoamericana de Ciencias Sociales Sede Ecuador (Ecuador)

Erwin Padua Xavier, Universidade Federal de Uberlândia (Brazil)

Esteban Arratia, Academia Nacional de Estudios Politicos y Estrategicos de Chile (Chile)

Eugênio Diniz, Pontifícia Universidade Católica de Minas Gerais (Brazil)

Evgenia Ilieva, Ithaca College (United States of America)

Fabiano Mielniczuk, Audiplo / Universidade Federal do Rio Grande do Sul (Brazil)

Fabiano Santos, Universidade do Estado do Rio de Janeiro (Brazil)

Fabio Albergaria de Queiroz, Universidade Católica de Brasília (Brazil)

Fabrício Chagas Bastos, Universidad de los Andes (Chile)

Federico Merke, Universidad de San Andrés (Argentina) 
Feliciano Sá Guimarães, Universidade de São Paulo (Brazil)

Felipe Kern Moreira, Universidade Federal do Rio Grande (Brazil)

Fernando Brancoli, Universidade Federal do Rio de Janeiro (Brazil)

Fidel Pérez Flores, Universidade de Brasília (Brazil)

Finn Laursen, University of Southern Denmark (Denmark)

Frank Mattheis, University of Pretoria (South Africa)

Gabriel Cepaluni, Universidade Estadual Paulista (Brazil)

Garrett Graddy-Lovelace, American University (United States of America)

Gelson Fonseca Júnior, Consulado-Geral do Brasil no Porto (Portugal)

Gilberto Rodrigues, Universidade Federal do $A B C$ (Brazil)

Giorgio Romano, Universidade Federal do ABC (Brazil)

Graciela de Conti Pagliari, Universidade Federal de Santa Catarina (Brazil)

Guilherme Casarões, Fundação Getúlio Vargas - São Paulo (Brazil)

Haroldo Ramanzini Jr., Universidade Federal de Uberlândia (Brazil)

Helen Kinsella, University of Winconsin-Madison (United States of America)

Heloise Weber, The University of Queensland (Australia)

Helton Ricardo Ouriques, Universidade Federal de Santa Catarina (Brazil)

Henrique Altemani, Pontifícia Universidade Católica de São Paulo (Brazil)

Henrique Furtado, University of Manchester (United Kingdom)

Hepzibah Muñoz Martínez, University of New Brunswick (Canada)

Himadeep Muppidi, Vassar College (United States of America)

Iara Costa Leite, Universidade Federal de Santa Catarina (Brazil)

Igor Abdalla, Pontifícia Universidade Católica do Rio de Janeiro (Brazil)

Isabel Rocha de Siqueira, Pontifícia Universidade Católica do Rio de Janeiro (Brazil)

Ivan Thiago de Oliveira, Instituto de Pesquisa Econômica Aplicada (Brazil)

Izabela Corrêa, London School of Economics and Political Science (United Kingdom)

Jakeet Singh, Illinois State University (United States of America)

Jakob Vestergaard, Danish Institute for International Studies (Denmark)

Jana Tabak, Pontifícia Universidade Católica do Rio de Janeiro (Brazil)

Janina Onuki, Universidade de São Paulo (Brazil)

Jasmin Habib, University of Waterloo (Canada)

Javier Vadell, Pontifícia Universidade Católica de Minas Gerais (Brazil)

Jean Daudelin, Carleton University (Canada)

Jean-Marie Chenou, Universidad de los Andes (Colombia)

Joakim Berndtsson, University of Gothenburg (Sweden)

João Henrique Roriz, Universidade Federal de Goiás (Brazil)

João Roberto Martins Filho, Universidade Federal de São Carlos (Brazil)

Joaquin Roy, University of Miami (United States of America)

Johannes Riber Nordby, Forsvaret Copenhagen (Denmark)

Jon Coaffee, University of Warwick (United Kingdom)

Jorge Battaglino, Universidad de Buenos Aires (Argentina) 
Jorge Lasmar, Pontifícia Universidade Católica de Minas Gerais (Brazil)

José Briceño-Ruiz, Universidad de los Andes (Colombia)

José Miguel Cruz, Florida International University (United States of America)

José Morandé, Universidad de Chile (Chile)

Joseph Marques, King's College London (United Kingdom)

Julian Gonzalez Guyer, Universidad de la República (Uruguay)

Juliana Farias, Universidade Estadual do Rio de Janeiro (Brazil)

Juliana Viggiano, Universidade Federal de Santa Catarina (Brazil)

Juliano Aragusuku, Universidade de Campinas (Brazil)

Juliet Johnson, McGill University (Canada)

Kai Kenkel, Pontifícia Universidade Católica do Rio de Janeiro (Brazil)

Kai Lehmann, Universidade de São Paulo (Brazil)

Kamal-Deen Ali, Centre for Maritime Law and Security Africa (Ghana)

Khatchik Der Ghougassian, Universidad de San Andrés (Argentina)

Klaus Dalgaard, Universidade de Campinas (Brazil)

Laura Horn, Roskilde University (Denmark)

Layla Dawood, Universidade do Estado do Rio de Janeiro (Brazil)

Leonardo Ramos, Pontifícia Universidade Católica de Minas Gerais (Brazil)

Letícia Carvalho, Fundação Getúlio Vargas-São Paulo (Brazil)

Letícia Pinheiro, Universidade do Estado do Rio de Janeiro (Brazil)

Lia Valls Pereira, Universidade do Estado do Rio de Janeiro (Brazil)

Lilian Burlamaqui Duarte, Ministério das Relações Exteriores (Brazil)

Lindy Heinecken, Stellenbosch Univeristy (South Africa)

Lisa Tilley, University of Warwick (United Kingdom)

Louiza Odysseos, University of Sussex (United Kingdom)

Lucas Melgaco, Vrije Universiteit Brussel (Belgium)

Lucas Pereira Rezende, Universidade Federal de Santa Catarina (Brazil)

Luis Leandro Schenoni, University of Notre Dame (United States of America)

Maíra Siman Gomes, Pontifícia Universidade Católica do Rio de Janeiro (Brazil)

Manaíra Assunção, Universität Hamburg (Germany)

Manfred Wilhelmy, Universidad de Chile (Chile)

Manoel Santos, Universidade Federal de Minas Gerais (Brazil)

Manuela Picq, Universidad San Francisco de Quito (Ecuador)

Manuela Trindade, Pontifícia Universidade Católica do Rio de Janeiro (Brazil)

Mara Tignino, University of Geneva (Switzerland)

Marcela Vecchione, Universidade Federal do Pará (Brazil)

Marcelo Coutinho, Fundação Getúlio Vargas-São Paulo (Brazil)

Marcelo Mariano, Universidade do Espírito Santo (Brazil)

Marcelo Medeiros, Universidade Federal de Pernambuco (Brazil)

Marcelo Nonnenberg, Pontifícia Universidade Católica do Rio de Janeiro (Brazil)

Marcelo Valença, Universidade do Estado do Rio de Janeiro (Brazil) 
Marcial Suarez, Universidade Federal Fluminense (Brazil)

Marco Aurélio Cepik, Universidade Federal do Rio Grande do Sul (Brazil)

Marcos Aurélio Guedes de Oliveira, Universidade Federal de Pernambuco (Brazil)

Maria Francisca Ramos de Gil Saraiva, Universidade de Lisboa (Portugal)

Maria Izabel Carvalho, Universidade de Brasília (Brazil)

Maria Luisa Mendonça, Universidade do Estado do Rio de Janeiro (Brazil)

Marianne Franklin, Goldsmiths, University of London (United Kingdom)

Marianne Wiesebron, Leiden Universit (Netherlands)

Mariano Barbato, Universität Passau (Germany)

Mario Rapoport, Universidad de Buenos Aires (Argentina)

Marta Chantal Ribeiro, Faculdade de Direito da Universidade do Porto (Portugal)

Marta Fernández, Pontifícia Universidade Católica do Rio de Janeiro (Brazil)

Mathieu Turgeon, Universidade de Brasília (Brazil)

Matias Margulis, University of Stirling (United Kingdom)

Matilde de Souza, Pontifícia Universidade Católica de Minas Gerais (Brazil)

Matt Davies, Newcastle University (United Kingdom)

Maureen Santos, Pontificia Universidade Católica do Rio de Janeiro (Brazil)

Maurício Santoro, Universidade do Estado do Rio de Janeiro (Brazil)

Michael Dillon, Lancaster University (United Kingdom)

Michael Shapiro, University of Hawai'i (United States of America)

Michael Strange, Malmö University (Sweden)

Miriam Santos, Universidade Federal Rural do Rio de Janeiro (Brazil)

Miriam Saraiva, Universidade do Estado do Rio de Janeiro (Brazil)

Monica Herz, Pontifícia Universidade Católica do Rio de Janeiro (Brazil)

Monica Salomon, Universidade Federal de Santa Catarina (Brazil)

Mustafa Murat Yurtbilir, Australian National University (Australia)

Mustapha Pasha, Aberystwyth University (United Kingdom)

Mvuselelo Ngcoya, University of KwaZulu-Natal (South Africa)

Naeem Inayatullah, Ithaca College (United States of America)

Narendran Kumarakulasingam, University of KwaZulu-Natal (South Africa)

Natália Félix, Pontifícia Universidade Católica de São Paulo (Brazil)

Noé Cornago, University of the Basque Country (Spain)

Norma Breda, Universidade de Brasília (Brazil)

Odete Maria de Oliveira, Universidade Comunitária da Região de Chapecó (Brazil)

Oliver Stuenkel, Fundação Getúlio Vargas-São Paulo (Brazil)

Paul Amar, University of California-Santa Barbara (United States of America)

Paul Kellog, Athabasca University (Canada)

Paula Drumond, Graduate Institute of International and Development Studies (Switzerland)

Paula Duarte Lopes, Universidade de Coimbra (Portugal)

Paulo Afonso Velasco, Universidade do Estado do Rio de Janeiro (Brazil)

Paulo Almeida, Ministério das Relações Exteriores (Brazil) 
Paulo Chamon, Pontifícia Universidade Católica do Rio de Janeiro (Brazil)

Paulo Manduca, Universidade de Campinas (Brazil)

Pedro Feliú Ribeiro, Universidade de São Paulo (Brazil)

Peter Vale, University of Johannesburg (South Africa)

Pia Riggirozzi, University of Southampton (United Kingdom)

Pierre Vercauteren, Université Catholique de Louvain (Belgium)

Pinar Bilgin, Bilkent University (Turkey)

Rafael Villa, Universidade de São Paulo (Brazil)

Randall Germain, Carleton University (Canada)

Raquel Vaz-Pinto, Universidade Nova de Lisboa (Portugal)

Ravi de Costa, York University (Canada)

Reginaldo Nasser, Pontifícia Universidade Católica de São Paulo (Brazil)

Reinaldo Dias, Universidade Presbiteriana Mackenzie (Brazil)

Renato Maluf, Universidade Federal Rural do Rio de Janeiro (Brazil)

Renato Petrocchi, Universidade Federal Fluminense (Brazil)

Ricardo Verdum, Universidade Federal de Santa Catarina (Brazil)

Rita Abrahamsen, University of Ottawa (Canada)

Robbie Shilliam, Queen Mary, University of London (United Kingdom)

Robert Muggah, Instituto Igarapé (Brazil)

Robert O'Brien, McMaster University (Canada)

Rodrigo Luiz Medeiros Silva, Universidade Federal da Integração Latino-Americana (Brazil)

Rogério de Souza Farias, University of Chicago (United States of America)

Rut Diamint, Universidad Torcuato Di Tella (Argentina)

Samson Opondo, Vassar College (United States of America)

Samuel Alves Soares, Universidade Estadual Paulista (Brazil)

Sanjay Seth, Goldsmiths, University of London (United Kingdom)

Sean Burges, Australian National University (Australia)

Sergio Luiz Cruz Aguilar, Universidade Estadual Paulista (Brazil)

Shiguenoli Miyamoto, Universidade de Campinas (Brazil)

Simon Springer, University of Victoria (Canada)

Simone Gomes, Universidade do Estado do Rio de Janeiro (Brazil)

Stefano Guzzini, Uppsala University (Sweden)

Sunil Dasgupta, University of Maryland (United States of America)

Thiago Lima, Universidade Federal da Paraíba (Brazil)

Timothy Sinclair, University of Warwick (United Kingdom)

Togzhan Kassenova, Carnegie Endowment for International Peace (United States of America)

Tomás Ayuso, Network of Researchers in International Affairs (United States of America)

Tony Porter, McMaster University (Canada)

Tullo Vigevani, Universidade Estadual Paulista (Brazil)

Vagner Alves, Universidade Federal Fluminense (Brazil)

Victor Coutinho Lage, Pontifícia Universidade Católica do Rio de Janeiro (Brazil) 
Vineet Thakur, University of Johannesburg (South Africa)

Vinícius de Carvalho, King's College London (United Kingdom)

William Coleman, University of Waterloo (Canada)

\section{CINT Communication Team}

Akta Kaushal, University of Hawaii (United States of America)

Benjamin de Carvalho, Norwegian Institute of International Affairs (Norway)

Daniel Aragão, Universidade Federal da Bahia (Brazil)

Daniel Castelan, Universidade Federal de Santa Catarina (Brazil)

Elif Kalaycioglu, University of Minnesota (United States of America)

Fabricio Chagas Bastos, Universidad de los Andes (Chile)

Henrique Tavares Furtado, University of Manchester (United Kingdom)

Jonathan Austin, The Graduate Institute of International and Development Studies (Switzerland)

Paula Duarte Lopes,Universidade de Coimbra (Portugal)

Paulo Chamon, Pontifícia Universidade Católica do Rio de Janeiro (Brazil)

Teresa Cravo, Universidade de Coimbra (Portugal) 